\title{
RELAÇÕES ENTRE AS COLONIZAÇÕES POR FUNGOS MICORRIZICOS ARBUSCULARES E TEORES DE NUTRIENTES FOLIARES EM OITO ESPÉCIES FLORESTAIS DA AMAZONIA
}

\author{
Luiz Antonio de OLIVEIRA ${ }^{1,3}$, Tibério Leonardo GUITTON ${ }^{2}$, Francisco Wesen \\ MOREIRA $^{1}$
}

RESUMO - Na Amazônia encontra-se um grande número de espécies florestais, muitas delas já com reconhecido valor econômico, como a Abiurana (Eremoluma williamii), Andiroba (Carapa guianensis), Cardeiro (Scleronema micranthum), Cedrorana (Cedrelinga catenaeformis), Cumarú (Dipterix odorata), Jacareúba (Calophyllum angulare), Marupá (Simaruba amara) e Piquiá (Caryocar villosum). Pouco se sabe sobre suas caracteristicas nutricionais e infecçòes por micorrizas arbusculares (MA) quando em solos ácidos e de baixa fertilidade da região. Com o objetivo de obter informações neste sentido, foi realizada uma avaliação de campo com estas espécies em fase adulta. Foram coletadas amostras de solos (rizosfera), raizes e folhas das espécies, de plantios experimentais na Estação Experimental de Silvicultura Tropical do INPA, Manaus, AM, em dois solos podzólicos. Todas as espécies apresentaram infecções por MA, mas diferiram quanto às colonizações, bem como nas concentrações de macro e micronutrientes nas folhas. Foram encontradas algumas correlações significativas (oito de um total de 64) entre as colonizações por micorrizas nas raizes e os teores de $\mathrm{Ca}, \mathrm{P}, \mathrm{Cu}, \mathrm{Fe}, \mathrm{Mn}$ e $\mathrm{Zn}$ nas folhas das espécies. Estas correlações estatisticamente significativas indicam que as endomicorrizas estão contribuindo para a absorção de nutrientes pelas plantas nas condições de terra firme da Amazônia Central.

Palavras chaves: Andiroba, Abiurana, Cardeiro, Cedrorana, Cumaru, Jacareúba, Marupá, Piquiá, nutrição de plantas, fertilidade do solo.

Relationship Between Arbuscular Mycorrhizae Fungi Colonization and leaf Nutrients in Eight Amazonian Forestry Species

ABSTRACT - A large number of forestry species are found in the Amazon, several of which are of economic value, such as Abiurana (Eremoluma williamii), Andiroba (Carapa guianensis), Cardeiro (Scleronema micranthum), Cedrorana (Cedrelinga catenaeformis), Cumarú (Dipterix odorata), Jacareúba (Calophyllum angulare), Marupá (Simaruba amara) and Piquiá (Caryocar villosum). Their nutritional requirements and arbuscular mycorrhizae (AM) affinity are generally unknown when growing on acid and low fertility soils. Root, soil and leaf samples from adult plants were collected from experimental plantations growing on ultisols near Manaus, AM, Brazil. All the species presented endomycorrhizal fungi, but differed in terms of colonization index, as well as macro and micronutrient contents of the leaves. Some statistically significant relationships were found (eight of a total of 64) between root colonization and $\mathrm{Ca}, \mathrm{P}, \mathrm{Cu}, \mathrm{Fe}, \mathrm{Mn}$ and $\mathrm{Zn}$ contents of the leaves. These relationships suggest that endomycorrhizae are contributing to plant nutrient absorption under the acid and low fertile soils in the Central Amazon.

Key words: Andiroba, Abiurana, Cardeiro, Cedrorana, Cumaru, Jacareúba, Marupá, Piquiá, plant nutrition, soil fertility.

INTRODUÇÃO

Na Amazônia encontra-se um grande número de espécies florestais, muitas com reconhecido valor econômico madeireiro, como a Abiurana (Eremoluma williamii Aubr. et Pellegr.), Andiroba (Carapa guianensis Aubl.), Cardeiro (Scleronema micranthum Ducke), Cedrorana (Cedrelinga catenaeformis

INPA, Coordenação de Pesq. em Cièncias Agronômicas - Caixa postal 478, 69011-970 Manaus - AM.

2 Confederação Nacional da Agricultura - Setor Bancário Norte - Quadra I - Bloco F - 70040800 - Brasilia - DF

3. Bolsista do CNPq. (Proc. № 521227/94.1 e 520814/96.7 (RE) 
Ducke), Cumarú (Dipterix odorata (Aubl.) Willd.), Jacareúba (Calophyllum angulare A.C. Smith), Marupá (Simaruba amara Aubl.) e Piquá (Caryocar villosum Aubl.). A extração de madeira na região é caracterizada pelo extrativismo por aniquilamento (Homma, 1993), o que vem causando problemas de ordem ecológica e econômica em muitas partes da região, onde várias espécies estão desaparecendo. A pesquisa florestal com espécies madeireiras tem um papel muito importante no sentido de preservá-las e manter o estoque regional, pois permite que as espécies sejam mais conhecidas quanto às suas caracteristicas, para serem posteriormente usadas em sistemas florestais com rendimento sustentado.

$\mathrm{Um}$ dos aspectos poucos estudados se refere à habilidade que as espécies têm de se adaptarem aos solos ácidos e de baixa fertilidade da Amazônia. Suas associações com microrganismos do solo, como as micorrizas arbusculares (MA), podem ser responsáveis por essa adaptação. Desde sua descoberta, as pesquisas com micorrizas têm demonstrado o efeito benéfico destas associações no estabelecimento e desenvolvimento de plantas em solos de baixa fertilidade (Schenck, 1984). No entanto, existem poucos estudos feitos com micorrizas arbusculares (MA) em espécies nativas da Amazônia, como os trabalhos realizados por St. John (1980 a;b;c), St. John \& Uhl (1983), Bonetti et al. (1984), que apenas reportam suas ocorrências, sem avaliar suas contribuições para a nutrição das plantas. Apenas a presença do fungo na raiz não significa contribuição na nutrição da planta, conforme documentado por diversos autores (Bonetti, 1984; Howeler et al., 1987; Saggin Júnior et al., 1995; Silveira et al., 1995), uma vez que a eficiência da simbiose pode variar, dependendo da planta, do fungo e do meio ambiente.

Com relação às espécies do presente estudo, pouco se sabe sobre suas características nutricionais e colonizações por fungos micorrízicos quando em solos ácidos e de baixa fertilidade da região, bem como se esta simbiose contribui, de algum modo, para melhorar os seus estados nutricionais. Este trabalho supre algumas destas informações, que poderão servir de base para futuros estudos de nutrição com as mesmas.

\section{MATERIAL E MÉTODOS}

\section{Material}

Foram avaliadas oito espécies florestais em dois plantios experimentais, localizados na Estação Experimental de Silvicultura Tropical do INPA (Instituto Nacional de Pesquisas da Amazônia), região de Manaus, $\mathrm{AM}$, Brasil ( $2^{\circ} 40^{\prime}$ Sul e $60^{\circ} 20^{\prime}$ Oeste), num Podzólico vermelho amarelo de classe textura média (área S1) e num Podzólico vermelho amarelo de textura barro argilo arenoso (área S6), segundo a classificação granulométrica de USDA, EUA (Ranzani, 1980). Estas parcelas de experimentação silvicultural (S1 e S6) foram implantadas pela Coordenação de Pesquisas em Silvicultura Tropical/INPA, sendo distantes uma da outra em torno de dois quilômetros.

As espécies estudadas na área S1 foram o Cumarú, Jacareúba e Marupá. 
As plantas são de um plantio a pleno sol, espaçadas entre si por $4 \times 4 \mathrm{~m}$, tendo sido plantadas em 12/72 (Cumarú), 02/73 (Jacareúba) e 01/73 (Marupá). Na área S6 foram pesquisadas as espécies Abiurana, Andiroba, Cardeiro, Cedrorana e Piquiá. As plantas se encontram em um plantio a pleno sol, espaçadas entre si por $3 \times 3 \mathrm{~m}$, ten.. sido plantadas em 04/78 (Abiurana, Andiroba, Cardeiro e Piquiá) e 05/78 (Cedrorana).

\section{MÉTODOS}

Foram coletadas para as análises, folhas e raizes em duas épocas (11/93 e 05/94), usando-se cinco plantas por espécie como repetições. As amostras de solos foram retiradas da rizosfera de cada planta, à profundidade de $0-20 \mathrm{~cm}$, sendo submetidas às análises físicas (textura, método da pipeta) (EMBRAPA, 1979) e químicas (Vettori, 1969; EMBRAPA, 1979) no Laboratório de Solos da Coordenação de Pesquisas em Ciências Agronômicas/ INPA. As análises químicas consistiram das seguintes metodologias: $\mathrm{pH}$ (água), $\mathrm{Ca}$, $\mathrm{Mg}$ e $\mathrm{Al}(\mathrm{KCl} 1 \mathrm{~N}), \mathrm{P}$ (extração pelo Mehlich 1 e leitura por colorimetria), $\mathrm{K}$, $\mathrm{Mn}, \mathrm{Zn}$ e Fe (Mehlich 1, absorção atômica) e, carbono pelo método de Walkley-Black. Os resultados dos solos das duas áreas se encontram na Tabela 1.

As folhas foram retiradas do terço superior da copa, segundo Van Den Driessche (1974) e separadas em três classes: das extremidades dos ramos (novas), intermediárias ou medianas (médias) e basais (velhas), conforme Dall'orto et al. (1976), formando uma amostra composta para as determinações químicas segundo
Tabela 1. Caracteristicas dos podzólicos amazônicos das áreas experimentais S1 e S6 da Estação Experimental de Silvicultura Tropical do INPA.

\begin{tabular}{lcc}
\hline $\begin{array}{l}\text { CARACTERISTICAS DOS } \\
\text { SOLOS }\end{array}$ & AREA S1 & AREA S6 \\
\hline $\mathrm{PH}\left(\mathrm{H}_{2} \mathrm{O}\right)$ & 3,8 & 3,9 \\
$\mathrm{P}\left(\mathrm{mg}^{\mathrm{k}} \mathrm{kg}^{-1}\right)$ & 3 & 3 \\
$\mathrm{~K}\left(\mathrm{mg}_{\mathrm{kg}}{ }^{-1}\right)$ & 21 & 45 \\
$\mathrm{Ca}\left(\mathrm{cmol}^{(+)} \cdot \mathrm{kg}^{-1}\right)$ & 0,35 & 0,34 \\
$\mathrm{Mg}\left(\mathrm{cmol}(+) \cdot \mathrm{kg}^{-1}\right)$ & 0,21 & 0,27 \\
$\mathrm{Al}\left(\mathrm{cmol}(+) \cdot \mathrm{kg}^{-1}\right)$ & 0,32 & 0,66 \\
$\mathrm{Mn}\left(\mathrm{mg}^{-1} \mathrm{~kg}^{-1}\right)$ & 10 & 5 \\
$\mathrm{Zn}\left(\mathrm{mg}^{-1} \mathrm{~kg}^{-1}\right)$ & 19 & 20 \\
$\mathrm{Fe}\left(\mathrm{mg} \cdot \mathrm{kg}^{\prime}\right)$ & 41 & 125 \\
$\mathrm{C}(\%)$ & 5,5 & 1,9 \\
Areia $(\%)$ & 82,4 & 57,8 \\
Silte $(\%)$ & 6,8 & 15,4 \\
Argila (\%) & 10,8 & 26,8 \\
\hline
\end{tabular}

EMBRAPA (1988). As colonizações pelos fungos micorrízicos nas raizes clarificadas com $\mathrm{KOH}$ e coloridas segundo Kormanick et al. (1980) foram avaliadas observando-as em lupa e microscópio. Realizou-se a análise estatística dos dados, bem como das correlações, segundo Gomez \& Gomez (1984) e Pimentel Gomes (1987), entre as ocorrências das micorrizas arbusculares e os teores de nutrientes em cada uma das espécies para verificar se esta associação planta-fungo influencia a absorção de nutrientes do solo. O delineamento experimental foi em blocos inteiramente casualizados e as correlações foram analisadas de forma pontual. Para isso correlacionou-se os teores de elementos das folhas de cada planta em cada época, com as ocorrências de micorrizas na mesma planta no dia de coleta dessas folhas, dando assim, para cada espécie em cada área, um total de dez amostragens (cinco plantas $\mathrm{x}$ duas épocas $=\mathrm{dez}$ pontos de correlações para cada espécie). 


\section{RESULTADOS E DISCUSSÃO}

\section{Área S1: Podzólico vermelho amarelo, textura média}

Houve diferenças entre as três espécies para várias das características analisadas, tais como infecções por MA e teores de $\mathrm{Ca}, \mathrm{Mg}, \mathrm{P}, \mathrm{Zn}$ e $\mathrm{Mn}$ (Tab. 2). Não houve diferenças entre as espécies apenas para os teores de $\mathrm{K}$, $\mathrm{Fe}$ e $\mathrm{Cu}$. O Marupá se destacou das demais para a maioria das características analisadas, exceto para os teores de $\mathrm{Zn}$ e Mn. Esta espécie apresentou as maiores porcentagens de colonizações por fungos micorrizicos, teores de $\mathrm{Ca}, \mathrm{Mg}, \mathrm{P}$ e, uma tendência de maior concentração de $\mathrm{K}$, embora sem diferença estatística. O Cumaru apresentou as menores infecções por MA e concentrações de $\mathrm{Ca}$ e $\mathrm{Mg}$, mas as maiores concentrações de $\mathrm{P}$.

Estas diferenças entre as espécies quanto aos teores de nutrientes nas folhas sugerem que elas apresentam diferentes habilidades para absorver estes nutrientes do solo ácido ou que apresentam variações nas eficiências de uso interno destes elementos. Neste último caso, menor concentração pode significar maior eficiência de uso, com conseqüente diluição nos tecidos, mas altas produções de biomassas. Menores habilidades de absorção, por outro lado, podem indicar que estejam mais próximas dos limites criticos destes elementos no solo, passivel de mostrarse deficientes dos mesmos com mais facilidade. Espécies com este perfil necessitariam, mais comumente, de uma adubação com estes nutrientes nos solos de baixa fertilidade de terra firmu da Amazônia (latossolos e podzólicos) (Clark, 1984; Kamprath, 1984). No entanto, como a fisiologia destas espécies é pouco connecida, deve-se efetuar estudos mais detalhados para se definir quais os limites críticos da concentração de cada um dos macro e micronutrientes nos seus tecidos, como forma de estabelecer critérios e necessidades de adubação para as mesmas. Estes estudos permitirão saber se as diferenças de concentrações de nutrientes nestas espécies são provenientes da variação da habilidade de absorção do solo ou da eficiência interna no uso destes elementos.

Quando se compara as concentrações dos macronutrientes (Tab. 2), observou-se que as de $\mathrm{K}$ foram sempre superiores às de $\mathrm{Ca}$, estando em desacordo com as afirmações de Curlin (1970), pelas quais a maioria das espécies florestais

Tabela 2. Porcentagem de infecções por micorrizas VA e composição mineral foliar das espécies estudadas na área S1. Médias de duas épocas de coletas, dez repetições.

\begin{tabular}{lccccccccc}
\hline & $\mathrm{MA}$ & $\mathrm{Ca}$ & $\mathrm{Mg}$ & $\mathrm{P}$ & $\mathrm{K}$ & $\mathrm{Fe}$ & $\mathrm{Zn}$ & $\mathrm{Mn}$ & $\mathrm{Cu}$ \\
\hline ESPÉCIES & $(\%)$ & $(\%)$ & $(\%)$ & $(\%)$ & $(\%)$ & $(\mathrm{mg} \cdot \mathrm{kg}-1)$ & $(\mathrm{mg} \cdot \mathrm{kg}-1)$ & $(\mathrm{mg} \cdot \mathrm{kg}-1)$ & $(\mathrm{mg} \cdot \mathrm{kg}-1)$ \\
Cumaru & $30,9 \mathrm{~b}$ & $0,09 \mathrm{~b}$ & $0,14 \mathrm{~b}$ & $0,09 \mathrm{a}$ & $0,37 \mathrm{a}$ & $81 \mathrm{a}$ & $26 \mathrm{a}$ & $61 \mathrm{a}$ & $5 \mathrm{a}$ \\
Jacareúba & $39,0 \mathrm{~b}$ & $0,16 \mathrm{a}$ & $0,16 \mathrm{ab}$ & $0,06 \mathrm{~b}$ & $0,35 \mathrm{a}$ & $97 \mathrm{a}$ & $34 \mathrm{a}$ & $19 \mathrm{c}$ & $6 \mathrm{a}$ \\
Marupá & $52,6 \mathrm{a}$ & $0,19 \mathrm{a}$ & $0,18 \mathrm{a}$ & $0,08 \mathrm{ab}$ & $0,42 \mathrm{a}$ & $92 \mathrm{a}$ & $8 \mathrm{~b}$ & $39 \mathrm{~b}$ & $7 \mathrm{a}$ \\
Médias & 40,8 & 0,15 & 0,16 & 0,08 & 0,38 & 90 & 23 & 40 & 6 \\
\hline
\end{tabular}

Obs.: As médias com as mesmas letras nas colunas não diferem estatisticamente pelo teste de Tukey ao nivel de $5 \%$ de probabilidade. 
acumula os nutrientes na ordem $\mathrm{Ca}>\mathrm{K}>$ P. Pela Tabela 2 observa-se que esta relação foi em geral, $\mathrm{K}>\mathrm{Mg}=\mathrm{Ca}>\mathrm{P}$, estando de acordo com os observados por Epstein (1975). A maior concentração do $\mathrm{K}$ em relação ao Ca talvez seja decorrente do baixo $\mathrm{pH}$, o que confirmaria as observações de Barbosa et al. (1995) de que em condições de $\mathrm{pH}$ baixo, o K sobrepuja os teores de $\mathrm{Ca}$, o que permitiria ainda detectar características calcícolas nas espécies estudadas.

Quanto aos micronutrientes, observou-se em geral a relação $\mathrm{Fe}>\mathrm{Mn}$ $>\mathrm{Zn}>\mathrm{Cu}$, estando em conformidade com as quantidades relativas encontradas por outros autores, como Epstein (1975), Soria et al. (1982) e Sarmiento et al. (1992). A
Jacareúba foi a mais eficiente na absorção de $\mathrm{Zn}$, com tendência de o ser também em Fe. O Marupá apresentou teor de $\mathrm{Zn}$ significativamente inferior ao encontrado nas outras espécies. Observa-se que somente os teores de Mn apresentaram diferenças marcantes entre as espécies estudadas na área $\mathrm{S} 1$. Os teores de $\mathrm{Fe}$ e $\mathrm{Cu}$ não apresentaram diferenças estatísticas entre as espécies.

Houve poucas correlações significativas entre as colonizações dos fungos micorrizicos arbusculares e os teores de nutrientes nas folhas das três espécies (Tab. 3). O Cumaru não apresentou correlações significativas com as características avaliadas, enquanto a Jacareúba apresentou três e o Marupá

Tabela 3. Correlações entre as colonizações por micorrizas arbusculares (MA) e os teores de macro e micronutrientes nas folhas das três espécies da área SI.

\begin{tabular}{lll}
\hline ESPÉCIES & \multicolumn{1}{c}{ EQUAÇŌES(1) $^{(1)}$} & VALORES DE r \\
\hline Cumaru & $\mathrm{Ca}=0,001 \mathrm{MA}+0,083$ & $0,225 \mathrm{~ns}^{(2)}$ \\
& $\mathrm{Mg}=0,001 \mathrm{MA}+0,124$ & $0,317 \mathrm{~ns}$ \\
$\mathrm{P}=0,001 \mathrm{MA}+0,085$ & $0,077 \mathrm{~ns}$ \\
& $\mathrm{~K}=0,002 \mathrm{MA}+0,310$ & $0,301 \mathrm{~ns}$ \\
& $\mathrm{Fe}=0,841 \mathrm{MA}+55,02$ & $0,550 \mathrm{~ns}$ \\
& $\mathrm{Zn}=-0,152 \mathrm{MA}+29,00$ & $0,325 \mathrm{~ns}$ \\
& $\mathrm{Mn}=-0,327 \mathrm{MA}+71,18$ & $0,308 \mathrm{~ns}$ \\
Jacareúba & $\mathrm{Cu}=0,107 \mathrm{MA}+1,801$ & $0,395 \mathrm{~ns}$ \\
& $\mathrm{Ca}=0,001 \mathrm{MA}+0,109$ & $0,438 \mathrm{~ns}$ \\
& $\mathrm{Mg}=-0,001 \mathrm{MA}+0,168$ & $0,066 \mathrm{~ns}$ \\
& $\mathrm{P}=0,001 \mathrm{MA}+0,048$ & $0,837^{*}$ \\
& $\mathrm{~K}=0,002 \mathrm{MA}+0,248$ & $0,566 \mathrm{~ns}$ \\
& $\mathrm{Fe}=-0,466 \mathrm{MA}+114,8$ & $0,643^{*}$ \\
& $\mathrm{Zn}=-0,673 \mathrm{MA}+60,82$ & $0,580 \mathrm{~ns}$ \\
& $\mathrm{Mn}=-0,107 \mathrm{MA}+23,76$ & $0,267 \mathrm{~ns}$ \\
& $\mathrm{Cu}=0,114 \mathrm{MA}+1,162$ & $0,801^{* *}$ \\
& $\mathrm{Ca}=0,001 \mathrm{MA}+0,135$ & $0,402 \mathrm{~ns}$ \\
& $\mathrm{Mg}=0,001 \mathrm{MA}+0,169$ & $0,075 \mathrm{~ns}$ \\
& $\mathrm{P}=0,001 \mathrm{MA}+0,059$ & $0,481 \mathrm{~ns}$ \\
& $\mathrm{~K}=0,001 \mathrm{MA}+0,409$ & $0,064 \mathrm{~ns}$ \\
& $\mathrm{Fe}=0,034 \mathrm{MA}+74,04$ & $0,034 \mathrm{~ns}$ \\
& $\mathrm{Zn}=0,114 \mathrm{MA}+2,097$ & $0,737^{*}$ \\
& $\mathrm{Mn}=-0,329 \mathrm{MA}+56,30$ & $0,409 \mathrm{~ns}$ \\
& $\mathrm{Cu}=0,092 \mathrm{MA}+2,742$ & $0,598 \mathrm{~ns}$
\end{tabular}

(1) - macronutrientes em \% e micronutrientes em mg.kg-1

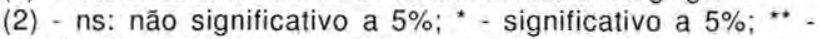
significativo a $1 \%$ de probabilidade. 
uma. Na Jacareúba, as correlações significativas foram com os nutrientes $\mathrm{P}$, $\mathrm{Cu}$ e $\mathrm{Fe}$, sendo positivas para os dois primeiros e negativa para o último, segundo os coeficientes das equações. Portanto, as micorrizas contribuiram para a concentração do $\mathrm{P}$ e Cu na Jacareúba, mas esta associação pode diminuir o teor de $\mathrm{Fe}$ pelas plantas, mantendo sua concentração semelhante à encontrada nas outras duas espécies.

Com relação ao Marupá, as micorrizas contribuíram apenas para a concentração do $\mathrm{Zn}$, embora seus teores nas plantas tenham sido os menores das três espécies estudadas (Tab. 2). Estes resultados realçam o fato de que embora o P seja ọ nutriente ao qual se dá maior importância nesta simbiose, por ter baixa concentração em solos intemperizados, pode ocorrer também uma grande absorção de outros nutrientes, especialmente $\mathrm{Ca}, \mathrm{Zn}, \mathrm{Cu}$ e S (Silveira, 1992). Apenas a Jacareúba apresentou relação significativa entre a concentração de $\mathrm{Pe}$ as colonizações por MA, estando portanto de acordo com muitos trabalhos que reportam esta correlação positiva (Lopes et al., 1983; Lynch, 1986; Siqueira \& Franco, 1988; Silveira, 1992).

\section{Área S6 : Podzólico Vermelho Amarelo textura barro argilo arenosa}

Nesta área, apenas os teores de $\mathrm{Ca} e$ $\mathrm{Cu}$ foram iguais estatisticamente entre as cinco espécies estudadas (Tab. 4). Todas as outras características analisadas apresentaram diferenças entre as espécies. Os maiores índices de colonizações por MA foram observados na Cedrorana e Andiroba, com a Abiurana apresentando os menores.

Quanto aos macronutrientes, os menores teores de $\mathrm{Mg}$ foram observados nas folhas de Piquiá $(0,23 \%)$; contudo este valor diferiu apenas em relação às concentrações alcançadas por Andiroba e Abiurana. A concentração de $\mathrm{P}$ obtida no tecido foliar de Cedrorana $(0,16 \%)$ foi, exceto quanto ao Cardeiro, significativamente superior a todas as demais espécies, o que ocorreu também quanto ao teor foliar de $\mathrm{K}$. Este acúmulo superior de $\mathrm{P}$ e $\mathrm{K}$ apresentado pela Cedrorana poderia ser decorrente da maior eficácia da simbiose MA, pois também apresentou as maiores taxas de colonização micorrízica. No entanto, a espécie não apresentou correlação positiva entre as variáveis (Tab. 5), talvez porque o fator eficiência

Tabela 4. Porcentagem de colonizações por micorrizas arbusculares (MA) e composição mineral foliar das espécies estudadas na área S6. Médias de duas épocas de coletas, dez repetições.

\begin{tabular}{llllllllll}
\hline & $\mathrm{MA}$ & $\mathrm{Ca}$ & $\mathrm{Mg}$ & $\mathrm{P}$ & $\mathrm{K}$ & $\mathrm{Fe}$ & $\mathrm{Zn}$ & $\mathrm{Mn}$ & $\begin{array}{c}\mathrm{Cu} \\
(\%) \\
\text { ESPÉCIES }\end{array}$ \\
$(\%)$ & $(\%)$ & $(\%)$ & $(\%)$ & $(\%)$ & $(\mathrm{mg} \cdot \mathrm{kg}-1)$ & $(\mathrm{mg} \cdot \mathrm{kg}-1)$ & $(\mathrm{mg} \cdot \mathrm{kg}-1)$ \\
\hline Abiurana & $25 \mathrm{~b}$ & $0,41 \mathrm{a}$ & $0,36 \mathrm{a}$ & $0,05 \mathrm{c}$ & $0,25 \mathrm{~d}$ & $80 \mathrm{~b}$ & $14 \mathrm{~b}$ & $27 \mathrm{c}$ & $7 \mathrm{a}$ \\
Andiroba & $50 \mathrm{a}$ & $0,32 \mathrm{a}$ & $0,36 \mathrm{a}$ & $0,08 \mathrm{bc}$ & $0,44 \mathrm{~cd}$ & $101 \mathrm{ab}$ & $16 \mathrm{~b}$ & $14 \mathrm{c}$ & $6 \mathrm{a}$ \\
Cardeiro & $44 \mathrm{ab}$ & $0,28 \mathrm{a}$ & $0,28 \mathrm{ab}$ & $0,11 \mathrm{ab}$ & $0,55 \mathrm{bc}$ & $111 \mathrm{ab}$ & $18 \mathrm{~b}$ & $135 \mathrm{a}$ & $7 \mathrm{a}$ \\
Cedrorana & $61 \mathrm{a}$ & $0,29 \mathrm{a}$ & $0,33 \mathrm{ab}$ & $0,16 \mathrm{a}$ & $0,89 \mathrm{a}$ & $102 \mathrm{ab}$ & $28 \mathrm{a}$ & $103 \mathrm{ab}$ & $5 \mathrm{a}$ \\
Piquiá & $47 \mathrm{ab}$ & $0,28 \mathrm{a}$ & $0,23 \mathrm{~b}$ & $0,09 \mathrm{~b}$ & $0,60 \mathrm{~b}$ & $123 \mathrm{a}$ & $13 \mathrm{~b}$ & $54 \mathrm{bc}$ & $8 \mathrm{a}$ \\
Médias & 45 & 0,32 & 0,32 & 0,10 & 0,55 & 103 & 18 & 67 & 7 \\
\hline
\end{tabular}

Obs.: As médias com as mesmas letras nas colunas nâo diferem estatisticamente pelo teste de Tukey ao nivel de $5 \%$ de probabilidade. 
da simbiose esteja atuando significativamente neste caso. Esta hipótese é reforçada por resultados encontrados na literatura mostrando que ocorrem diferenças significativas de rendimento das plantas, mesmo quando os níveis das colonizações são semelhantes (Bonetti, 1984; Howeler et al., 1987; Saggin Júnior et al., 1995; Silveira et al., 1995).

De um modo geral, a acumulação total de macronutrientes apresentou a seguinte ordem decrescente: $\mathrm{K}>\mathrm{Ca}=$ $\mathrm{Mg}>\mathrm{P}$, sendo esta relação, semelhante à da área $\mathrm{S} 1$ e a apresentada por Epstein (1975). As concentrações de K foram sempre superiores às de $\mathrm{Ca}$, com exceção na Abiurana, onde a ordem de acúmulo de nutrientes seguiu o padrão observado por Kramer \& Kolowski (1960) e Curlin (1970), ou seja: $\mathrm{Ca}>\mathrm{K}>\mathrm{P}$. Isto sugere, quando comparados às médias apresentadas por Epstein (1975) uma possivel deficiência para estes nutrientes. Os valores médios mostrados por este autor foram: $\mathrm{K}=1,0 \%, \mathrm{Ca}=0,5 \% \mathrm{e}, \mathrm{Mg}$ ou $\mathrm{P}=0,2 \%$, sendo portanto, superiores aos da Tabela 4.

Com relação aos teores dos micronutrientes, o Piquiá apresentou maior concentração de Fe que a Abiurana, enquanto que em relação ao $\mathrm{Zn}$, a Cedrorana foi a que apresentou as maiores concentrações nas folhas. Finalmente, o Cardeiro foi a espécie que apresentou maiores concentrações de $\mathrm{Mn}$, não diferindo estatisticamente da Cedrorana. Das cinco espécies estudadas, a Abiurana foi a que apresentou as menores concentrações de $\mathrm{Fe}, \mathrm{Zn}$ e $\mathrm{Mn}$, sugerindo que ela apresenta menor capacidade de absorção destes nutrientes do solo e/ou que a mesma possui maior eficiência de conversão fotossintética, o que causaria uma diluição destes elementos nos tecidos vegetais. De um modo geral, as concentrações dos micronutrientes foram semelhantes às encontradas por Epstein (1975): $\mathrm{Fe}>\mathrm{Mn}>\mathrm{Zn}>\mathrm{Cu}$. A seqüência de acúmulo dos micronutrientes apresentou a seguinte conformação:

Fe: Piquiä $>=$ Cardeiro $>=$ Cedrorana $>=$ Andiroba $>=$ Abiurana
Mn: Cardeiro $>=$ Cedrorana $>=$ Piquia $>=$ Abiurana $>=$ Andiroba
$\mathrm{Zn}$ : Cedrorana $>$ Cardeiro $>=$ Andiroba $>=$ Abiurana $>=$ Piquiá
Cu: Piquia $>=$ Cardeiro $=$ Abiurana $>=$ Andiroba $>=$ Cedrorana

O acúmulo total de micronutrientes para cada espécie apresentou a seguinte ordem decrescente:
Abiurana: $\mathrm{Fe}>\mathrm{Mn}>\mathrm{Zn}>\mathrm{Cu}$
Andiroba: $\mathrm{Fe}>\mathrm{Zn}>\mathrm{Mn}>\mathrm{Cu}$
Cardeiro: $\mathrm{Mn}>\mathrm{Fe}>\mathrm{Zn}>\mathrm{Cu}$
Cedrorana: $\mathrm{Mn}>\mathrm{Fe}>\mathrm{Zn}>\mathrm{Cu}$
Piquiá: $\mathrm{Fe}>\mathrm{Mn}>\mathrm{Zn}>\mathrm{Cu}$

Observa-se que no Cardeiro e na Cedrorana, os teores de Mn superaram os de $\mathrm{Fe}$, diferindo da condição fisiológica $\mathrm{Fe}$ $>\mathrm{Mn}>\mathrm{Zn}$ já observada por vários autores (Epstein, 1975; Soria et al., 1982; Sarmiento et al., 1992). Estas seqüências de concentrações de macro e micronutrientes podem ser muito importantes numa possível indicação de espécies para sistemas agroflorestais, pois aquelas que apresentam seqüências iguais ou semelhantes poderão competir mais entre si ao serem colocadas na mesma área de solo, o que não seria o mais adequado. Numa escolha com base nestas características, o mais aconselhável seria escolher espécies que tendem a competir menos pelos mesmos nutrientes do solo, apresentando, portanto, seqüências diferentes. Partindo desta premissa e com base nas seqüências de micronutrientes acima, pode-se esperar maior competição entre a Abiurana e Piquiá, ou entre 
Cardeiro e Cedrorana, do que entre Andiroba e Cardeiro/Cedrorana.

Apenas quatro das correlações entre as concentrações dos macro e micronutrientes com as colonizações micorrizicas se mostraram estatisticamente significativas (Tab. 5), indicando que outros fatores relacionados com as espécies de plantas, fungos miconizicos, meio ambiente e a interação entre eles prevaleceram na maioria dos casos. No entanto, os poucos casos significativos encontrados comprovam que as micorrizas influenciaram na concentração do $\mathrm{P}$ e $\mathrm{Cu}$ na Andiroba e, $\mathrm{Ca}$ e $\mathrm{Mn}$ no Piquiá.

$\mathrm{Na}$ Andiroba, a taxa de colonização

Tabela 5. Correlações entre as colonizações por micorrizas arbusculares (MA) e os teores de macro e micronutrientes nas folhas das espécies da área S6.

\begin{tabular}{|c|c|c|}
\hline ESPÉCIES & EQUAÇŌES (1) & VALORES DE r \\
\hline \multirow[t]{8}{*}{ Abiurana } & $\mathrm{Ca}=0,002 \mathrm{MA}+0,342$ & $0,261 \mathrm{~ns}^{(2)}$ \\
\hline & $\mathrm{Mg}=0,003 \mathrm{MA}+0,282$ & $0,489 \mathrm{~ns}$ \\
\hline & $P=-0,001 M A+0,057$ & $0,149 \mathrm{~ns}$ \\
\hline & $K=-0,003 M A+0,341$ & $0,582 \mathrm{~ns}$ \\
\hline & $\mathrm{Fe}=0,383 \mathrm{MA}+71,65$ & $0,210 \mathrm{~ns}$ \\
\hline & $\mathrm{Zn}=0,001 \mathrm{MA}+13,58$ & $0,004 \mathrm{~ns}$ \\
\hline & $\mathrm{Mn}=0,289 \mathrm{MA}+19,38$ & $0,328 \mathrm{~ns}$ \\
\hline & $\mathrm{Cu}=0,072 \mathrm{MA}+5,41$ & $0,493 \mathrm{~ns}$ \\
\hline \multirow[t]{8}{*}{ Andiroba } & $\mathrm{Ca}=0,002 \mathrm{MA}+0,231$ & $0,266 \mathrm{~ns}$ \\
\hline & $M g=0,003 M A+0,231$ & $0,436 \mathrm{~ns}$ \\
\hline & $P=-0,001 M A+0,101$ & $0,669^{*}$ \\
\hline & $K=-0,005 \mathrm{MA}+0,693$ & $0,508 \mathrm{~ns}$ \\
\hline & $\mathrm{Fe}=-0,222 \mathrm{MA}+111,65$ & $0,175 \mathrm{~ns}$ \\
\hline & $Z n=0,116 M A+9,275$ & $0,426 \mathrm{~ns}$ \\
\hline & $M n=-0,137 M A+20,39$ & 0,449 ns \\
\hline & $\mathrm{Cu}=0,074 \mathrm{MA}+2,273$ & $0,640^{*}$ \\
\hline \multirow[t]{8}{*}{ Cardeiro } & $\mathrm{Ca}=0,001 \mathrm{MA}+0,241$ & $0,384 \mathrm{~ns}$ \\
\hline & $\mathrm{Mg}=0,001 \mathrm{MA}+0,269$ & $0,082 n s$ \\
\hline & $P=-0,001 M A+0,133$ & $0,396 n s$ \\
\hline & $K=-0,001 \mathrm{MA}+0,564$ & 0,049 ns \\
\hline & $\mathrm{Fe}=0,631 \mathrm{MA}+83,65$ & $0,525 \mathrm{~ns}$ \\
\hline & $Z n=0,081 M A+14,26$ & $0,433 \mathrm{~ns}$ \\
\hline & $M n=0,125 M A+129,99$ & $0,089 \mathrm{~ns}$ \\
\hline & $\mathrm{Cu}=-0,011 \mathrm{MA}+6,996$ & $0,145 \mathrm{~ns}$ \\
\hline \multirow[t]{8}{*}{ Cedrorana } & $\mathrm{Ca}=0,001 \mathrm{MA}+0,221$ & $0,097 \mathrm{~ns}$ \\
\hline & $\mathrm{Mg}=0,002 \mathrm{MA}+0,204$ & $0,339 \mathrm{~ns}$ \\
\hline & $P=-0,001 M A+0,162$ & 0,069 ns \\
\hline & $\mathrm{K}=-0,006 \mathrm{MA}+1,239$ & $0,327 \mathrm{~ns}$ \\
\hline & $\mathrm{Fe}=0,824 \mathrm{MA}+51,54$ & $0,549 \mathrm{~ns}$ \\
\hline & $Z n=0,114 M A+21,20$ & $0,189 \mathrm{~ns}$ \\
\hline & $\mathrm{Mn}=0,999 \mathrm{MA}+42,75$ & $0,201 \mathrm{~ns}$ \\
\hline & $\mathrm{Cu}=-0,105 \mathrm{MA}+11,58$ & $0,424 \mathrm{~ns}$ \\
\hline \multirow[t]{8}{*}{ Piquiá } & $\mathrm{Ca}=0,004 \mathrm{MA}+0,072$ & $0,675^{\star}$ \\
\hline & $M g=-0,001 M A+0,289$ & $0,344 \mathrm{~ns}$ \\
\hline & $P=-0,001 M A+0,117$ & $0,329 \mathrm{~ns}$ \\
\hline & $\mathrm{K}=-0,003 \mathrm{MA}+0,756$ & $0,308 \mathrm{~ns}$ \\
\hline & $\mathrm{Fe}=0,393 \mathrm{MA}+104,56$ & $0,243 \mathrm{~ns}$ \\
\hline & $Z n=0,030 M A+11,55$ & $0,099 \mathrm{~ns}$ \\
\hline & $M n=0,617 \mathrm{MA}+24,24$ & $0,654^{*}$ \\
\hline & $\mathrm{Cu}=0,032 \mathrm{MA}+6,868$ & $0,352 \mathrm{~ns}$ \\
\hline
\end{tabular}

(1) - macronutrientes em \% e micronutrientes em mg.kg-1

(2) - ns: năo significativo a $5 \%$; * - significativo a $5 \%$ de probabilidade. 
por MA apresentou correlação negativa com a concentração foliar de P. Tal resultado parece divergir dos beneficios proporcionados pelas MA em termos de crescimento e nutrição, amplamente documentados para muitas espécies vegetais, incluindo várias florestais (Maronek et al., 1981; Silva \& Siqueira, 1991; Weber \& Amorim, 1994). Contudo, a eficiência do processo de absorção e translocamento decorrente da simbiose está também estreitamente relacionada ao teor de fósforo disponivel, que embora não provoque necessariamente diferenças na capacidade de colonização do sistema radicular do hospedeiro, pode proporcionar diferenças quanto à efetividade na produção de matéria seca, no acúmulo de nutrientes e no índice de utilização de P (Sylvia \& Schenck, 1983; Shubert \& Hayman, 1986). Assim, a relação linear significativa e inversa observada na Andiroba pode ser consequêencia de uma melhor utilização do $\mathrm{P}$ na parte aérea, como já foi observado por Araújo et al. (1994) em tomateiro cultivado sob diferentes niveis de $\mathrm{P}$ no solo.

A relação linear significativa $\mathrm{e}$ positiva entre a colonização por MA e as concentrações de Ca no tecido foliar do Piquiá (Tab. 5), realça as observações de Silveira (1992) de que, embora o P seja o mais importante nutriente envolvido na resposta de crescimento das plantas micorrizadas, estas apresentam maior absorção de outros nutrientes, especialmente $\mathrm{Ca}, \mathrm{Zn}$, Cu e S, indicando a ocorrência de efeitos nutricionais benéficos decorrentes da simbiose.

Assim, as correlações significativas encontradas no presente trabalho comprovam que as micorrizas arbusculares contribuem para o balanço de nutrientes nas plantas em condições de campo na Amazônia, fato este sugerido por outros autores (St. John, 1980 a, b,c; St. John \& Uhl, 1983; Bonetti et al., 1984; Bonetti \& Navarro, 1990), mas sem esta comprovação.

\section{Bibliografia citada}

Araújo, A.P.; Silva, E. M. R.; Almeida, D. 1994. Efetividade de fungos endomicorrizicos em tomateiro em diferentes níveis de fósforo no solo. R. bras. Ci. Solo, 18(2):193-200.

Barbosa, Z.; Venturin, R.P.; Carvalho, J.G.; Morais, A.R. 1995. Crescimento e composição química foliar de mudas de aroeira (Myracruodon urundeuva (Fr. All.) Eng.) sob diferentes saturações por bases. II. Teor foliar de macronutrientes. In: Resumos ernandidos. XXV Congresso Brasileiro de Ciència do Solo, UFV, Viçosa, p.809-10.

Bonetti, R. 1984. Effect of vesicular-arbuscular mycorrhiza in the nodulation, growth and phosphorus and nitrogen uptake in siratro. R. bras. Ci. Solo, 8:189-192.

Bonetti, R.; Navarro, R.B. 1990. Ocorrência de micorriza vesículo-arbuscular (MVA) em espécies frutiferas nativas da região amazônica. Energ. Nucl. Agric, 11(1):26-33.

Bonetti, R.; Oliveira, L.A.; Magalhães, F.M.M. 1984. População de Rhizobium spp. e ocorrência de micorrizas VA em cultivos de essências florestais. Pesq. agropec. Bras., 19:137-142.

Clark, R.B. 1984. Physiological aspects of calcium, magnesium, and molybdenium deficiencies in plants. In: Adams, F. (ed.). Soil acidity and liming. $2^{\text {nd }}$ ed. ASA, Madison, Wisc., USA. p.99-170.

Curlin, J.W. 1970. Nutrient cycling as a factor in site productivity and forest fertilization. In: Youngberger, C.T.; Davey, C.B. (eds.). Tree growth and forest soils. Oregon Univ. Press, Corvallis. p.313-335.

Dall'orto, F.A.C.; Garlipp, R.C.D.; Brauner, L.J.; Miranda, M.T. 1976. Concentraçōes de alguns macro e micronutrientes em essências florestais do parque da Escola Superior de 
Agricultura Luiz de Queiroz. Anais da E.S.A.

"Luiz de Queiroz", 33:233-242.

EMBRAPA. 1979. Manual de métodos de análise de solos. Serviço Nacional de Levantamento e Conservação do Solo. EMBRAPA, Rio de Janeiro. 220p.

EMBRAPA. 1988. Análise foliar: laboratório de análises de solos e plantas. Centro Nacional de Pesquisas de Seringueira e Dendê, Manaus. $8 \mathrm{p}$.

Epstein, 1975. Nutrição mineral das plantas: principios e perspectivas. EDUSP, São Paulo, $341 \mathrm{p}$.

Gomez, K.A.; Gomez, A.A. 1984. Statistical procedures for agricultural research. $2^{\text {nd }}$ ed. John Wiley \& Sons, New York. 680p.

Homma, A.K.O. 1993. Extrativismo vegetal na Amazōnia: limites e oportunidades. EMBRAPA, Brasilia, DF. 202p.

Howeler, R.H.; Sieverding, E.; Saif, S. 1987. Practical aspects of mycorrhizal technology in some tropical crops and pastures. Plant and Soil, 100:249-283.

Kamprath, E.J. 1984. Crop response to lime on soils in the tropics. In: Adams, F. (ed.). Soil acidity and liming. $2^{\text {nd }}$ ed., ASA, Madison, Wisc., USA. p.349-368.

Kormanick, P.P.; Bryan, W.C.; Schultz, R.C. 1980. Procedures and equipment for staining large numbers of plant root samples for endomycorrhizal assay. Can. J. Microbiol., 26:536-538.

Kramer, P.J.; Kolowski, T. 1960. Fisiologia das árvores. Fundação C. Gulbenkian, Lisboa. $745 \mathrm{p}$.

Lopes, E.S.; Siqueira, J.O.; Zambolim, L. 1983. Caracterização das micorrizas vesicular-arbuscular (MVA) e seus efeitos no crescimento das plantas. $R$. bras. $C i$. Solo, 7:1-19.

Lynch, J.M. 1986. Biotecnologia do solo: fatores microbiológicos na produtividade agricola. Edit. Manole Ltda, São Paulo, 209p.

Maronek, D.M.; Hendrix, J.W.; Kierman, J. 1981. Mycorrhizal fungi and their importance in horticultural crop production. Hort. Rev, 3:172-213.

Pimentel Gomes, F. 1987. Curso de estatistica experimental. 12." ed., ESALQ, USP, Piracicaba. 467p.

Ranzani, G. 1980. Identificação e caracterização de alguns solos da Estação Experimental de Silvicultura Tropical. Acta Amazonica, 10(1):7-41.

Saggin Júnior, O.J.; Siqueira, J.O.; Guimarães, P.T.G.; Oliveira, E. 1995. Colonização do cafeciro por diferentes fungos micorrizicos: efeitos na formação das mudas e no crescimento em solo fumigado. R. bras. Ci. Solo, 19:213-220.

Sarmiento, J.L.; Garcia, J.L.; Grande, M.C.; Villalon, M.C.; Castro, A.; Mazuelos, C, 1992. Niveles criticos y equilibrios optimos de macronutrientes en la fertilización diferenciada de la vid. Suelos y Planta, 2(4):663-673.

Schenck, N.C. 1984. Methods and principles of mycorrhizal research. The American Phytopatology Society, St. Paul. 234p.

Shubert, A.; Hayman, D.S. 1986. Plant growth responses to vesicular-arbuscular mycorrhiza. XVI. Effectiveness of different endophytes at different levels of soil phosphate. New Phytol., 103:79-90.

Silva, L.F.C.; Siqueira, J.O. 1991. Crescimento e teores de nutrientes de mudas de abacateiro, mangueira e mamoeiro sob influência de diferentes espécies de fungos micorrizicos vesículo-arbusculares. $R$. bras. Ci, Solo, 15(3):283-288.

Silveira, A.P.D. 1992. Micorrizas. In: Cardoso, E.J.B.N.; Tsai, S.M.; Neves, M.C.P. (eds.) Microbiologia do Solo. Sociedade Brasileira de Ciência do Solo, Campinas. p.257-282.

Silveira, A.P.D.; Freitas, S.S.; Silva, L.R.C.; Lombardi, M.L.C.O.; Cardoso, E.J.B.N. 1995. Interações de micorrizas arbusculares e rizobactérias promotoras do crescimento em plantas de feijão. R. bras. Ci. Solo, 19:205-211.

Siqueira, J.O.; Franco, A.A. 1988. Biotecnologia do solo: fundamentos e perspectivas. MECESAL-FAEPE-ABEAS, Brasilia. 235p.

Soria, J.T.; Palacios, S.J.; Esteban, E. 1982. Estudio de la nutrición del almendro. Niveles criticos y equilibrados óptimos de macro y micronutrientes. An. Edafol. Agrobiol., 41:1003-1025. 
St. John, T.V. 1980a. A survey of micorrhizal infection in an Amazonian rain forest. Acta Amazonica, 10(3):527-533.

St. John, T.V. 1980b. Tamanho da raiz, pêlos radiculares e infecção por micorrizas: um reexame da hipótese de Baylis com árvores tropicais. Acta Amazonica, 10(1):141-145.

St. John, T.V. 1980c. Uma lista de espécies de plantas tropicais brasileiras naturalmente infectadas com micorriza vesiculararbuscular. Acta Amazonica, 10(1):229-234.

St. John, T.V.; Uhl, C. 1983. Mycorrhizae in the rain forest at San Carlos de Rio Negro, Venezuela. Acta Cient. Venezoelana, 34:233-237.

Sylvia, D.M.; Shenck, N.C. 1983. Application of superphosphate to mycorrhizal plants stimulates sporulation of phosphorustolerant vesicular-arbuscular mycorrhizal fungi. New Phytol., 95:655-661.

Van Den Driessche, H.K. 1974. Prediction of mineral nutrient status of trees by foliar analysis. The Bot. Ver: 40(3):347-394.

Vettori, L. 1969. Métodos de análises de solos. Boletim Técnico 7. Ministério da Agricultura, Rio de Janeiro. 24p.

Weber, O.B.; Amorim, M.C. 1994. Adubação fosfática e inoculação de fungos micorrizicos vesiculo-arbusculares em mamoeiro 'Solo', $R$. bras. Ci. Solo, 18(2):187-192. 